\title{
Integrability Formulas. Part I
}

\author{
Bo Li \\ Qingdao University of Science \\ and Technology \\ China
}

\author{
$\mathrm{Na} \mathrm{Ma}$ \\ Qingdao University of Science \\ and Technology \\ China
}

Summary. In this article, we give several differentiation and integrability formulas of special and composite functions including the trigonometric function, and the polynomial function.

MML identifier: INTEGR12, version: $\underline{7.11 .04 \quad 4.130 .1076}$

The papers [12], [2], [3], [1], [7], [11], [13], [4], [17], [8], [9], [6], [18], [5], [10], [15], [16], and [14] provide the terminology and notation for this paper.

One can check that there exists a subset of $\mathbb{R}$ which is closed-interval.

For simplicity, we use the following convention: $a, b, x, r$ are real numbers, $n$ is an element of $\mathbb{N}, A$ is a closed-interval subset of $\mathbb{R}, f, g, f_{1}, f_{2}, g_{1}, g_{2}$ are partial functions from $\mathbb{R}$ to $\mathbb{R}$, and $Z$ is an open subset of $\mathbb{R}$.

We now state a number of propositions:

(1) Suppose $Z \subseteq \operatorname{dom}\left(\frac{1}{f_{1}+f_{2}}\right)$ and for every $x$ such that $x \in Z$ holds $f_{1}(x)=1$ and $f_{2}=\square^{2}$. Then $\frac{1}{f_{1}+f_{2}}$ is differentiable on $Z$ and for every $x$ such that $x \in Z$ holds $\left(\frac{1}{f_{1}+f_{2}}\right)^{\prime}{ }_{Y Z}^{\prime}(x)=-\frac{2 \cdot x}{\left(1+x^{2}\right)^{2}}$.

(2) Suppose that $A \subseteq Z$ and $f=\frac{\frac{1}{g_{1}+g_{2}}}{f_{2}}$ and $f_{2}=$ the function $\operatorname{arccot}$ and $Z \subseteq$ ] $-1,1\left[\right.$ and $g_{2}=\square^{2}$ and for every $x$ such that $x \in Z$ holds $g_{1}(x)=1$ and $f_{2}(x)>0$ and $Z=\operatorname{dom} f$. Then $\int_{A} f(x) d x=(-($ the function $\ln ) \cdot($ the function $\operatorname{arccot}))(\sup A)-$ $(-($ the function $\ln ) \cdot($ the function $\operatorname{arccot}))(\inf A)$.

(3) Suppose that

(i) $A \subseteq Z$, 
(ii) for every $x$ such that $x \in Z$ holds (the function exp) $(x)<1$ and $f_{1}(x)=1$,

(iii) $Z \subseteq \operatorname{dom}(($ the function $\arctan ) \cdot($ the function $\exp ))$,

(iv) $Z=\operatorname{dom} f$, and

(v) $f=\frac{\text { the function } \exp }{f_{1}+(\text { the function } \exp )^{2}}$.

Then $\int_{A} f(x) d x=(($ the function $\arctan ) \cdot($ the function $\exp ))(\sup A)-$ $(($ the function $\arctan ) \cdot($ the function $\exp ))(\inf A)$.

(4) Suppose that

(i) $A \subseteq Z$,

(ii) for every $x$ such that $x \in Z$ holds (the function $\exp )(x)<1$ and $f_{1}(x)=1$,

(iii) $Z \subseteq \operatorname{dom}($ (the function arccot) $\cdot($ the function $\exp )$ ),

(iv) $Z=\operatorname{dom} f$, and

(v) $f=\frac{- \text { the function } \exp }{f_{1}+(\text { the function } \exp )^{2}}$.

Then $\int_{A} f(x) d x=(($ the function arccot $) \cdot($ the function $\exp ))(\sup A)-(($ the function arccot) $\cdot($ the function $\exp ))(\inf A)$.

(5) Suppose that

(i) $A \subseteq Z$,

(ii) $Z=\operatorname{dom} f$, and

(iii) $f=\left(\right.$ the function exp) $\frac{\text { the function } \sin }{\text { the function cos }}+\frac{\text { the function exp }}{(\text { the function cos) })^{2}}$.

Then $\int_{A} f(x) d x=(($ the function $\exp )$ (the function $\left.\tan )\right)(\sup A)-(($ the function exp) (the function $\tan ))(\inf A)$.

(6) Suppose that

(i) $A \subseteq Z$,

(ii) $Z=\operatorname{dom} f$, and

(iii) $f=\left(\right.$ the function exp) $\frac{\text { the function cos }}{\text { the function sin }}-\frac{\text { the function exp }}{\text { (the function } \sin )^{2}}$.

Then $\int_{A} f(x) d x=(($ the function $\exp )$ (the function $\left.\cot )\right)(\sup A)-(($ the function exp) (the function $\cot ))(\inf A)$.

(7) Suppose that

(i) $A \subseteq Z$,

(ii) for every $x$ such that $x \in Z$ holds $f_{1}(x)=1$,

(iii) $Z \subseteq]-1,1[$,

(iv) $Z=\operatorname{dom} f$, and

(v) $f=($ the function $\exp$ ) (the function $\arctan )+\frac{\text { the function exp }}{f_{1}+\square^{2}}$. 
Then $\int_{A} f(x) d x=(($ the function exp) $($ the function $\arctan ))(\sup A)-(($ the function $\exp )($ the function $\arctan ))(\inf A)$.

(8) Suppose that

(i) $A \subseteq Z$,

(ii) for every $x$ such that $x \in Z$ holds $f_{1}(x)=1$,

(iii) $Z \subseteq]-1,1[$,

(iv) $Z=\operatorname{dom} f$, and

(v) $f=\left(\right.$ the function exp) (the function arccot) $-\frac{\text { the function exp }}{f_{1}+\square^{2}}$.

Then $\int_{A} f(x) d x=(($ the function $\exp )$ (the function $\left.\operatorname{arccot})\right)(\sup A)-(($ the function $\exp )($ the function $\operatorname{arccot}))(\inf A)$.

(9) Suppose $A \subseteq Z=\operatorname{dom} f$ and $f=(($ the function $\exp ) \cdot($ the function $\sin ))$ (the function cos). Then $\int_{A} f(x) d x=(($ the function $\exp ) \cdot($ the function $\sin ))(\sup A)-(($ the function $\exp ) \cdot($ the function $\sin ))(\inf A)$.

(10) Suppose $A \subseteq Z=\operatorname{dom} f$ and $f=$ ((the function $\operatorname{exp)~}$ (the function $\cos )$ ) (the function $\sin$ ).

Then $\int_{A} f(x) d x=(-($ the function $\exp ) \cdot($ the function $\cos ))(\sup A)-$ $(-($ the function $\exp ) \cdot($ the function $\cos ))(\inf A)$.

(11) Suppose $A \subseteq Z$ and for every $x$ such that $x \in Z$ holds $x>0$ and $Z=\operatorname{dom} f$ and $f=(($ the function $\cos ) \cdot($ the function $\ln )) \frac{1}{\mathrm{id} Z}$. Then $\int_{A} f(x) d x=(($ the function sin $) \cdot($ the function $\ln ))(\sup A)-(($ the function $\sin ) \cdot($ the function $\ln ))(\inf A)$.

(12) Suppose $A \subseteq Z$ and for every $x$ such that $x \in Z$ holds $x>0$ and $Z=\operatorname{dom} f$ and $f=$ ((the function $\sin ) \cdot($ the function $\ln )$ ) $\frac{1}{\operatorname{id} Z}$. Then $\int_{A} f(x) d x=(-($ the function $\cos ) \cdot($ the function $\ln ))(\sup A)-$ $(-($ the function $\cos ) \cdot($ the function $\ln ))(\inf A)$.

(13) Suppose $A \subseteq Z=\operatorname{dom} f$ and $f=$ (the function $\exp$ ) ((the function cos) -(the function $\exp ))$. Then $\int_{A} f(x) d x=(($ the function sin $) \cdot($ the function $\exp ))(\sup A)-(($ the function sin $) \cdot($ the function $\exp ))(\inf A)$.

(14) Suppose $A \subseteq Z=\operatorname{dom} f$ and $f=$ (the function exp) ((the function $\sin$ ) $\cdot($ the function exp)).

Then $\int_{A} f(x) d x=(-($ the function $\cos ) \cdot($ the function $\exp ))(\sup A)-$ $(-($ the function $\cos ) \cdot($ the function $\exp ))(\inf A)$. 
(15) Suppose that $A \subseteq Z \subseteq \operatorname{dom}\left((\right.$ the function $\left.\ln ) \cdot\left(f_{1}+f_{2}\right)\right)$ and $r \neq 0$ and for every $x$ such that $x \in Z$ holds $g(x)=\frac{x}{r}$ and $g(x)>-1$ and $g(x)<1$ and $f_{1}(x)=1$ and $f_{2}=\left(\square^{2}\right) \cdot g$ and $Z=\operatorname{dom} f$ and $f=$ (the function arctan) $\cdot g$. Then $\int f(x) d x=\left(\operatorname{id}_{Z}((\right.$ the function $\arctan ) \cdot g)-\frac{r}{2}(($ the function $\ln )$ $\left.\left.\cdot\left(f_{1}+f_{2}\right)\right)\right)(\sup A)-\left(\operatorname{id}_{Z}((\right.$ the function $\arctan ) \cdot g)-\frac{r}{2}(($ the function $\ln )$ $\left.\left.\cdot\left(f_{1}+f_{2}\right)\right)\right)(\inf A)$.

(16) Suppose that $A \subseteq Z \subseteq \operatorname{dom}\left((\right.$ the function $\left.\ln ) \cdot\left(f_{1}+f_{2}\right)\right)$ and $r \neq 0$ and for every $x$ such that $x \in Z$ holds $g(x)=\frac{x}{r}$ and $g(x)>-1$ and $g(x)<1$ and $f_{1}(x)=1$ and $f_{2}=\left(\square^{2}\right) \cdot g$ and $Z=\operatorname{dom} f$ and $f=$ (the function arccot) $\cdot g$. Then $\int_{A} f(x) d x=\left(\operatorname{id}_{Z}((\right.$ the function $\operatorname{arccot}) \cdot g)+\frac{r}{2}(($ the function $\ln )$ $\left.\left.\cdot\left(f_{1}+f_{2}\right)\right)\right)(\sup A)-\left(\operatorname{id}_{Z}((\right.$ the function $\operatorname{arccot}) \cdot g)+\frac{r}{2}(($ the function $\ln )$ $\left.\left.\cdot\left(f_{1}+f_{2}\right)\right)\right)(\inf A)$.

(17) Suppose that

(i) $A \subseteq Z$,

(ii) $f=($ the function $\arctan ) \cdot f_{1}+\frac{\mathrm{id}_{Z}}{r\left(g+f_{1}^{2}\right)}$,

(iii) for every $x$ such that $x \in Z$ holds $g(x)=1$ and $f_{1}(x)=\frac{x}{r}$ and $f_{1}(x)>-1$ and $f_{1}(x)<1$,

(iv) $Z=\operatorname{dom} f$, and

(v) $f$ is continuous on $A$.

Then $\int_{A} f(x) d x=\left(\operatorname{id}_{Z}\left((\right.\right.$ the function $\left.\left.\arctan ) \cdot f_{1}\right)\right)(\sup A)-\left(\operatorname{id}_{Z}((\right.$ the function $\left.\left.\arctan ) \cdot f_{1}\right)\right)(\inf A)$.

(18) Suppose that

(i) $A \subseteq Z$,

(ii) $f=\left(\right.$ the function arccot) $\cdot f_{1}-\frac{\mathrm{id}_{Z}}{r\left(g+f_{1}^{2}\right)}$,

(iii) for every $x$ such that $x \in Z$ holds $g(x)=1$ and $f_{1}(x)=\frac{x}{r}$ and $f_{1}(x)>-1$ and $f_{1}(x)<1$,

(iv) $Z=\operatorname{dom} f$, and

(v) $f$ is continuous on $A$.

Then $\int_{A} f(x) d x=\left(\operatorname{id}_{Z}\left((\right.\right.$ the function $\left.\left.\operatorname{arccot}) \cdot f_{1}\right)\right)(\sup A)-\left(\operatorname{id}_{Z}((\right.$ the function arccot $\left.\left.) \cdot f_{1}\right)\right)(\inf A)$.

(19) Suppose that $A \subseteq Z \subseteq]-1,1[$ and for every $x$ such that $x \in Z$ holds $f_{1}(x)=1$ and $Z=\operatorname{dom} f$ and $Z \subseteq \operatorname{dom}\left(\left(\square^{n}\right) \cdot(\right.$ the function $\left.\arcsin )\right)$ and $1<n$ and $f=\frac{n\left(\left(\square^{n-1}\right) \cdot(\text { the function arcsin })\right)}{\left(\square^{\frac{1}{2}}\right) \cdot\left(f_{1}-\square^{2}\right)}$. Then $\int_{A} f(x) d x=\left(\left(\square^{n}\right) \cdot\right.$ (the function $\arcsin ))(\sup A)-\left(\left(\square^{n}\right) \cdot(\right.$ the function $\left.\arcsin )\right)(\inf A)$.

(20) Suppose that $A \subseteq Z \subseteq]-1,1[$ and for every $x$ such that $x \in Z$ holds 
$f_{1}(x)=1$ and $Z \subseteq \operatorname{dom}\left(\left(\square^{n}\right) \cdot(\right.$ the function $\left.\arccos )\right)$ and $Z=\operatorname{dom} f$ and $1<n$ and $f=\frac{n\left(\left(\square^{n-1}\right) \cdot(\text { the function arccos })\right)}{\left(\square^{\frac{1}{2}}\right) \cdot\left(f_{1}-\square^{2}\right)}$. Then $\int_{A} f(x) d x=$ $\left(-\left(\square^{n}\right) \cdot(\right.$ the function $\left.\arccos )\right)(\sup A)-\left(-\left(\square^{n}\right) \cdot(\right.$ the function $\left.\arccos )\right)$ $(\inf A)$.

(21) Suppose $A \subseteq Z$ and for every $x$ such that $x \in Z$ holds $f_{1}(x)=1$ and $Z \subseteq]-1,1[$ and $Z=\operatorname{dom} f$ and $f=$ (the function $\arcsin )+\frac{\operatorname{id} Z}{\left(\square^{\frac{1}{2}}\right) \cdot\left(f_{1}-\square^{2}\right)}$. Then $\int_{A} f(x) d x=\left(\operatorname{id}_{Z}(\right.$ the function $\left.\arcsin )\right)(\sup A)-\left(\operatorname{id}_{Z}\right.$ (the function $\arcsin ))(\inf A)$.

(22) Suppose $A \subseteq Z$ and for every $x$ such that $x \in Z$ holds $f_{1}(x)=1$ and $Z \subseteq]-1,1[$ and $Z=\operatorname{dom} f$ and $f=$ (the function $\arccos )-\frac{\operatorname{id} Z}{\left(\square^{\frac{1}{2}}\right) \cdot\left(f_{1}-\square^{2}\right)}$. Then $\int_{A} f(x) d x=\left(\operatorname{id}_{Z}(\right.$ the function $\left.\arccos )\right)(\sup A)-\left(\operatorname{id}_{Z}\right.$ (the function $\arccos ))(\inf A)$.

(23) Suppose that

(i) $A \subseteq Z$,

(ii) $Z \subseteq]-1,1[$,

(iii) for every $x$ such that $x \in Z$ holds $f_{1}(x)=a \cdot x+b$ and $f_{2}(x)=1$,

(iv) $Z=\operatorname{dom} f$, and

(v) $f=a$ (the function $\arcsin )+\frac{f_{1}}{\left(\square^{\frac{1}{2}}\right) \cdot\left(f_{2}-\square^{2}\right)}$.

Then $\int_{A} f(x) d x=\left(f_{1}(\right.$ the function $\left.\arcsin )\right)(\sup A)-\left(f_{1}\right.$ (the function $\arcsin ))(\inf A)$.

(24) Suppose that

(i) $A \subseteq Z$,

(ii) $Z \subseteq]-1,1[$,

(iii) for every $x$ such that $x \in Z$ holds $f_{1}(x)=a \cdot x+b$ and $f_{2}(x)=1$,

(iv) $Z=\operatorname{dom} f$, and

(v) $f=a$ (the function $\arccos )-\frac{f_{1}}{\left(\square^{\frac{1}{2}}\right) \cdot\left(f_{2}-\square^{2}\right)}$.

Then $\int_{A} f(x) d x=\left(f_{1}\right.$ (the function $\left.\left.\arccos \right)\right)(\sup A)-\left(f_{1}\right.$ (the function $\arccos ))(\inf A)$.

(25) Suppose that

(i) $A \subseteq Z$,

(ii) for every $x$ such that $x \in Z$ holds $g(x)=1$ and $f_{1}(x)=\frac{x}{a}$ and $f_{1}(x)>-1$ and $f_{1}(x)<1$,

(iii) $Z=\operatorname{dom} f$,

(iv) $f$ is continuous on $A$, and 
(v) $f=($ the function $\arcsin ) \cdot f_{1}+\frac{\mathrm{id}_{Z}}{a\left(\left(\square^{\frac{1}{2}}\right) \cdot\left(g-f_{1}^{2}\right)\right)}$.

Then $\int_{A} f(x) d x=\left(\operatorname{id}_{Z}\left((\right.\right.$ the function $\left.\left.\arcsin ) \cdot f_{1}\right)\right)(\sup A)-\left(\operatorname{id}_{Z}((\right.$ the function $\left.\left.\arcsin ) \cdot f_{1}\right)\right)(\inf A)$

(26) Suppose that

(i) $A \subseteq Z$,

(ii) for every $x$ such that $x \in Z$ holds $g(x)=1$ and $f_{1}(x)=\frac{x}{a}$ and $f_{1}(x)>-1$ and $f_{1}(x)<1$,

(iii) $Z=\operatorname{dom} f$,

(iv) $f$ is continuous on $A$, and

(v) $f=($ the function $\arccos ) \cdot f_{1}-\frac{\operatorname{id}_{z}}{a\left(\left(\square^{\frac{1}{2}}\right) \cdot\left(g-f_{1}^{2}\right)\right)}$.

Then $\int_{A} f(x) d x=\left(\operatorname{id}_{Z}\left((\right.\right.$ the function $\left.\left.\arccos ) \cdot f_{1}\right)\right)(\sup A)-\left(\operatorname{id}_{Z}((\right.$ the function $\left.\left.\arccos ) \cdot f_{1}\right)\right)(\inf A)$.

(27) Suppose $A \subseteq Z$ and $f=\frac{n\left(\left(\square^{n-1}\right) \cdot(\text { the function } \sin )\right)}{\left(\square^{n+1}\right) \cdot(\text { the function } \cos )}$ and $1 \leq n$ and $Z \subseteq$ $\operatorname{dom}\left(\left(\square^{n}\right) \cdot(\right.$ the function $\left.\tan )\right)$ and $Z=\operatorname{dom} f$. Then $\int f(x) d x=\left(\left(\square^{n}\right) \cdot\right.$ (the function $\tan ))(\sup A)-\left(\left(\square^{n}\right) \cdot(\right.$ the function $\left.\tan )\right)(\inf A)$.

(28) Suppose $A \subseteq Z$ and $f=\frac{n\left(\left(\square^{n-1}\right) \cdot(\text { the function } \cos )\right)}{\left(\square^{n+1}\right) \cdot(\text { the function } \sin )}$ and $1 \leq n$ and $Z \subseteq \operatorname{dom}\left(\left(\square^{n}\right) \cdot(\right.$ the function $\left.\cot )\right)$ and $Z=\operatorname{dom} f$. Then $\int_{A} f(x) d x=$ $\left(-\left(\square^{n}\right) \cdot(\right.$ the function $\left.\cot )\right)(\sup A)-\left(-\left(\square^{n}\right) \cdot(\right.$ the function $\left.\cot )\right)(\inf A)$.

(29) Suppose that

(i) $A \subseteq Z$,

(ii) $Z \subseteq \operatorname{dom}\left((\right.$ the function $\left.\tan ) \cdot f_{1}\right)$,

(iii) $f=\frac{\left((\text { the function } \sin ) \cdot f_{1}\right)^{2}}{\left.(\text { (the function } \cos ) \cdot f_{1}\right)^{2}}$,

(iv) for every $x$ such that $x \in Z$ holds $f_{1}(x)=a \cdot x$ and $a \neq 0$, and

(v) $Z=\operatorname{dom} f$.

Then $\int f(x) d x=\left(\frac{1}{a}\left((\right.\right.$ the function $\left.\left.\tan ) \cdot f_{1}\right)-\operatorname{id}_{Z}\right)(\sup A)-\left(\frac{1}{a}((\right.$ the function $\left.\left.\tan ) \cdot f_{1}\right)-\operatorname{id}_{Z}\right)(\inf A)$.

(30) Suppose that

(i) $A \subseteq Z$,

(ii) $Z \subseteq \operatorname{dom}\left((\right.$ the function $\left.\cot ) \cdot f_{1}\right)$,

(iii) $f=\frac{\left((\text { the function } \cos ) \cdot f_{1}\right)^{2}}{\left.(\text { (the function } \sin ) \cdot f_{1}\right)^{2}}$,

(iv) for every $x$ such that $x \in Z$ holds $f_{1}(x)=a \cdot x$ and $a \neq 0$, and

(v) $Z=\operatorname{dom} f$. 
Then $\int_{A} f(x) d x=\left(\left(-\frac{1}{a}\right)\left((\right.\right.$ the function $\left.\left.\cot ) \cdot f_{1}\right)-\operatorname{id}_{Z}\right)(\sup A)-\left(\left(-\frac{1}{a}\right)((\right.$ the function cot) $\left.\left.\cdot f_{1}\right)-\operatorname{id}_{Z}\right)(\inf A)$.

(31) Suppose that

(i) $A \subseteq Z$,

(ii) for every $x$ such that $x \in Z$ holds $f_{1}(x)=a \cdot x+b$,

(iii) $Z=\operatorname{dom} f$, and

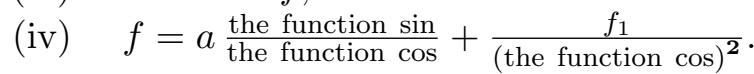

Then $\int_{A} f(x) d x=\left(f_{1}(\right.$ the function $\left.\tan )\right)(\sup A)-\left(f_{1}(\right.$ the function tan $\left.)\right)(\inf A)$.

(32) Suppose that

(i) $A \subseteq Z$,

(ii) for every $x$ such that $x \in Z$ holds $f_{1}(x)=a \cdot x+b$,

(iii) $Z=\operatorname{dom} f$, and

(iv) $f=a \frac{\text { the function } \cos }{\text { the function } \sin }-\frac{f_{1}}{{\text { (the function } \sin )^{2}}^{2}}$.

Then $\int_{A} f(x) d x=\left(f_{1}(\right.$ the function $\left.\cot )\right)(\sup A)-\left(f_{1}(\right.$ the function $\left.\cot )\right)(\inf A)$.

(33) Suppose that

(i) $A \subseteq Z$,

(ii) for every $x$ such that $x \in Z$ holds $f(x)=\frac{\text { (the function } \sin )(x)^{2}}{(\text { the function } \cos )(x)^{2}}$,

(iii) $Z \subseteq \operatorname{dom}($ (the function $\left.\tan )-\mathrm{id}_{Z}\right)$,

(iv) $Z=\operatorname{dom} f$, and

(v) $f$ is continuous on $A$.

Then $\int_{A} f(x) d x=\left((\right.$ the function $\left.\tan )-\mathrm{id}_{Z}\right)(\sup A)-(($ the function $\left.\tan )-\mathrm{id}_{Z}\right)(\inf A)$.

(34) Suppose that

(i) $A \subseteq Z$,

(ii) for every $x$ such that $x \in Z$ holds $f(x)=\frac{\text { (the function } \cos )(x)^{2}}{(\text { the function } \sin )(x)^{2}}$,

(iii) $Z \subseteq \operatorname{dom}\left(-\right.$ the function $\cot -\mathrm{id}_{Z}$ ),

(iv) $Z=\operatorname{dom} f$, and

(v) $f$ is continuous on $A$.

Then $\int_{A} f(x) d x=\left(\right.$-the function $\left.\cot -\operatorname{id}_{Z}\right)(\sup A)-($-the function $\cot -$ $\left.\operatorname{id}_{Z}\right)(\inf A)$.

(35) Suppose that

(i) $A \subseteq Z$,

(ii) for every $x$ such that $x \in Z$ holds $f(x)=\frac{1}{x \cdot\left(1+(\text { the function } \ln )(x)^{2}\right)}$ and (the function $\ln )(x)>-1$ and (the function $\ln )(x)<1$, 
(iii) $Z \subseteq \operatorname{dom}(($ the function $\arctan ) \cdot($ the function $\ln ))$,

(iv) $Z=\operatorname{dom} f$, and

(v) $f$ is continuous on $A$.

Then $\int_{A} f(x) d x=(($ the function $\arctan ) \cdot($ the function $\ln ))(\sup A)-(($ the function $\arctan ) \cdot($ the function $\ln ))(\inf A)$.

(36) Suppose that

(i) $A \subseteq Z$,

(ii) for every $x$ such that $x \in Z$ holds $f(x)=-\frac{1}{x \cdot\left(1+(\text { the function } \ln )(x)^{2}\right)}$ and (the function $\ln )(x)>-1$ and (the function $\ln )(x)<1$,

(iii) $Z \subseteq \operatorname{dom}(($ the function arccot) $\cdot($ the function $\ln ))$,

(iv) $Z=\operatorname{dom} f$, and

(v) $f$ is continuous on $A$.

Then $\int_{A} f(x) d x=(($ the function arccot $) \cdot($ the function $\ln ))(\sup A)-(($ the function arccot) $\cdot($ the function $\ln ))(\inf A)$.

(37) Suppose that

(i) $A \subseteq Z$,

(ii) for every $x$ such that $x \in Z$ holds $f(x)=\frac{a}{\sqrt{1-(a \cdot x+b)^{2}}}$ and $f_{1}(x)=a \cdot x+b$ and $f_{1}(x)>-1$ and $f_{1}(x)<1$,

(iii) $Z \subseteq \operatorname{dom}\left((\right.$ the function $\left.\arcsin ) \cdot f_{1}\right)$,

(iv) $Z=\operatorname{dom} f$, and

(v) $f$ is continuous on $A$.

Then $\int_{A} f(x) d x=\left((\right.$ the function $\left.\arcsin ) \cdot f_{1}\right)(\sup A)-(($ the function $\arcsin )$ $\left.\cdot f_{1}\right)(\inf A)$.

(38) Suppose that

(i) $A \subseteq Z$,

(ii) for every $x$ such that $x \in Z$ holds $f(x)=\frac{a}{\sqrt{1-(a \cdot x+b)^{2}}}$ and $f_{1}(x)=a \cdot x+b$ and $f_{1}(x)>-1$ and $f_{1}(x)<1$,

(iii) $\quad Z \subseteq \operatorname{dom}\left((\right.$ the function $\left.\arccos ) \cdot f_{1}\right)$,

(iv) $Z=\operatorname{dom} f$, and

(v) $f$ is continuous on $A$.

Then $\int_{A} f(x) d x=\left(-(\right.$ the function $\left.\arccos ) \cdot f_{1}\right)(\sup A)-(-($ the function $\left.\arccos ) \cdot f_{1}\right)(\inf A)$.

(39) Suppose that $A \subseteq Z$ and $f_{1}=g-f_{2}$ and $f_{2}=\square^{2}$ and for every $x$ such that $x \in Z$ holds $f(x)=x \cdot\left(1-x^{2}\right)^{-\frac{1}{2}}$ and $g(x)=1$ and $f_{1}(x)>0$ and $Z \subseteq \operatorname{dom}\left(\left(\square^{\frac{1}{2}}\right) \cdot f_{1}\right)$ and $Z=\operatorname{dom} f$ and $f$ is continuous on $A$. Then $\int_{A} f(x) d x=$ 
$\left(-\left(\square^{\frac{1}{2}}\right) \cdot f_{1}\right)(\sup A)-\left(-\left(\square^{\frac{1}{2}}\right) \cdot f_{1}\right)(\inf A)$.

(40) Suppose that $A \subseteq Z$ and $g=f_{1}-f_{2}$ and $f_{2}=\square^{2}$ and for every $x$ such that $x \in Z$ holds $f(x)=x \cdot\left(a^{2}-x^{2}\right)^{-\frac{1}{2}}$ and $f_{1}(x)=a^{2}$ and $g(x)>0$ and $Z \subseteq \operatorname{dom}\left(\left(\square^{\frac{1}{2}}\right) \cdot g\right)$ and $Z=\operatorname{dom} f$ and $f$ is continuous on $A$. Then $\int_{A} f(x) d x=$ $\left(-\left(\square^{\frac{1}{2}}\right) \cdot g\right)(\sup A)-\left(-\left(\square^{\frac{1}{2}}\right) \cdot g\right)(\inf A)$.

(41) Suppose that

(i) $A \subseteq Z$,

(ii) $n>0$,

(iii) for every $x$ such that $x \in Z$ holds $f(x)=\frac{(\text { the function } \cos )(x)}{(\text { the function } \sin )(x)^{n+1}}$ and (the function $\sin )(x) \neq 0$,

(iv) $Z \subseteq \operatorname{dom}\left(\left(\square^{n}\right) \cdot \frac{1}{\text { the function sin }}\right)$,

(v) $Z=\operatorname{dom} f$, and

(vi) $f$ is continuous on $A$.

Then $\int_{A} f(x) d x=\left(\left(-\frac{1}{n}\right)\left(\left(\square^{n}\right) \cdot \frac{1}{\text { the function } \sin }\right)\right)(\sup A)-\left(\left(-\frac{1}{n}\right)\left(\left(\square^{n}\right)\right.\right.$. $\left.\left.\frac{1}{\text { the function } \sin }\right)\right)(\inf A)$.

(42) Suppose that

(i) $A \subseteq Z$,

(ii) $n>0$,

(iii) for every $x$ such that $x \in Z$ holds $f(x)=\frac{\text { (the function } \sin )(x)}{(\text { the function } \cos )(x)^{n+1}}$ and (the function $\cos )(x) \neq 0$,

(iv) $Z \subseteq \operatorname{dom}\left(\left(\square^{n}\right) \cdot \frac{1}{\text { the function cos }}\right)$,

(v) $Z=\operatorname{dom} f$, and

(vi) $\quad f$ is continuous on $A$.

Then $\int_{A} f(x) d x=\left(\frac{1}{n}\left(\left(\square^{n}\right) \cdot \frac{1}{\text { the function } \cos }\right)\right)(\sup A)-\left(\frac{1}{n}\left(\left(\square^{n}\right)\right.\right.$. $A$
1

$\overline{\text { the function } \cos }))(\inf A)$.

(43) Suppose that $A \subseteq Z$ and $f=\frac{\frac{1}{g_{1}+g_{2}}}{f_{2}}$ and $f_{2}=$ the function arccot and $Z \subseteq$ ]-1,1[ and $g_{2}=\square^{2}$ and for every $x$ such that $x \in Z$ holds $f(x)=\frac{1}{\left(1+x^{2}\right) \cdot(\text { the function } \operatorname{arccot})(x)}$ and $g_{1}(x)=1$ and $f_{2}(x)>0$ and $Z=$ $\operatorname{dom} f$. Then $\int_{A} f(x) d x=(-($ the function $\ln ) \cdot($ the function $\operatorname{arccot}))(\sup A)-$ $(-($ the function $\ln ) \cdot($ the function $\operatorname{arccot}))(\inf A)$.

(44) Suppose that

(i) $A \subseteq Z$,

(ii) $Z \subseteq]-1,1[$,

(iii) for every $x$ such that $x \in Z$ holds (the function $\arcsin )(x)>0$ and $f_{1}(x)=1$, 
(iv) $Z \subseteq \operatorname{dom}(($ the function $\ln ) \cdot($ the function $\arcsin ))$,

(v) $Z=\operatorname{dom} f$, and

(vi) $\quad f=\frac{1}{\left(\left(\square^{\frac{1}{2}}\right) \cdot\left(f_{1}-\square^{2}\right)\right) \text { (the function arcsin) }}$.

Then $\int_{A} f(x) d x=(($ the function $\ln ) \cdot($ the function $\arcsin ))(\sup A)-(($ the function $\ln ) \cdot($ the function $\arcsin ))(\inf A)$.

(45) Suppose that

(i) $A \subseteq Z$,

(ii) $Z \subseteq]-1,1[$,

(iii) for every $x$ such that $x \in Z$ holds $f_{1}(x)=1$ and (the function $\left.\arccos \right)(x)>0$,

(iv) $Z \subseteq \operatorname{dom}(($ the function $\ln ) \cdot($ the function $\arccos ))$,

(v) $Z=\operatorname{dom} f$, and

(vi) $\quad f=\frac{1}{\left(\left(\square^{\frac{1}{2}}\right) \cdot\left(f_{1}-\square^{2}\right)\right)(\text { the function arccos) }}$.

Then $\int_{A} f(x) d x=(-($ the function $\ln ) \cdot($ the function $\arccos ))(\sup A)-$

$(-($ the function $\ln ) \cdot($ the function $\arccos ))(\inf A)$.

\section{REFERENCES}

[1] Czesław Byliński. Partial functions. Formalized Mathematics, 1(2):357-367, 1990.

[2] Noboru Endou and Artur Korniłowicz. The definition of the Riemann definite integral and some related lemmas. Formalized Mathematics, 8(1):93-102, 1999.

[3] Noboru Endou, Katsumi Wasaki, and Yasunari Shidama. Definition of integrability for partial functions from $\mathbb{R}$ to $\mathbb{R}$ and integrability for continuous functions. Formalized Mathematics, 9(2):281-284, 2001.

[4] Krzysztof Hryniewiecki. Basic properties of real numbers. Formalized Mathematics, 1(1):35-40, 1990.

[5] Artur Korniłowicz and Yasunari Shidama. Inverse trigonometric functions arcsin and arccos. Formalized Mathematics, 13(1):73-79, 2005.

[6] Jarosław Kotowicz. Convergent real sequences. Upper and lower bound of sets of real numbers. Formalized Mathematics, 1(3):477-481, 1990.

[7] Jarosław Kotowicz. Partial functions from a domain to a domain. Formalized Mathematics, 1(4):697-702, 1990.

[8] Jarosław Kotowicz. Partial functions from a domain to the set of real numbers. Formalized Mathematics, 1(4):703-709, 1990.

[9] Jarosław Kotowicz. Real sequences and basic operations on them. Formalized Mathematics, 1(2):269-272, 1990.

[10] Xiquan Liang and Bing Xie. Inverse trigonometric functions arctan and arccot. Formalized Mathematics, 16(2):147-158, 2008, doi:10.2478/v10037-008-0021-3.

[11] Konrad Raczkowski. Integer and rational exponents. Formalized Mathematics, 2(1):125$130,1991$.

[12] Konrad Raczkowski and Paweł Sadowski. Real function continuity. Formalized Mathematics, 1(4):787-791, 1990.

[13] Konrad Raczkowski and Paweł Sadowski. Topological properties of subsets in real numbers. Formalized Mathematics, 1(4):777-780, 1990.

[14] Yasunari Shidama. The Taylor expansions. Formalized Mathematics, 12(2):195-200, 2004.

[15] Andrzej Trybulec and Czesław Byliński. Some properties of real numbers. Formalized Mathematics, 1(3):445-449, 1990.

[16] Zinaida Trybulec. Properties of subsets. Formalized Mathematics, 1(1):67-71, 1990. 
[17] Edmund Woronowicz. Relations defined on sets. Formalized Mathematics, 1(1):181-186, 1990.

[18] Yuguang Yang and Yasunari Shidama. Trigonometric functions and existence of circle ratio. Formalized Mathematics, 7(2):255-263, 1998.

Received November 7, 2009 